\title{
Title: Evaluation of the diagnostic values and utility of Helico- bacter pylori stool antigen lateral immunochromatography
}

\author{
assay
}

\author{
Shaymaa Abdelmalek ${ }^{1 *}$, Wafy Hamed ${ }^{3}$, Neven Nagy ${ }^{1}$, Karim Shokry $^{1}$, and Hisham Abdelrahman ${ }^{2}$ \\ 1 1.Microbiology, Immunology Department, Faculty of Veterinary medicine, Cairo University, Egypt. Shay- \\ maa malek@cu.edu.eg, neveennagywaheeb@yahoo.com, karimshokry97@gmail.com \\ 2 Department of Veterinary Hygiene and Management, Faculty of Veterinary Medicine, Cairo University, \\ Egypt. hisham@auburn.edu \\ 3 Microbiology Department, Faculty of Veterinary Medicine, Sadat City University, Sadat City, Egypt. \\ Wafy_hamed@yahoo.com \\ * Correspondence: E.mail: shaymaa malek@cu.edu.eg, s.abdelmalek@nu.edu.eg, Phone: (+2)01220669524 \\ ORCID: 0000-0002-3333-7502, Postal code: 11221
}

\begin{abstract}
Background: Helicobacter pylori is the most common human gastric infection. H. pylori stool antigen lateral flow immunochromatography assay (HpSA-LFIA) is considered one of the most cost-effective and rapid non-invasive assays (active tests). The evaluation of this test is crucial for accuracy and utility assurance. This study aimed to evaluate the polyclonal antibody-based HpSA-LFIA in comparison to a monoclonal antibody-based ELISA kit.
\end{abstract}

Methodology: Stool samples were collected from 200 gastric patients for HpSA-LFIA and semiquantitative HpSA-ELISA. Statistical analysis of the diagnostic values was performed using MedCalc software. Chi-square tests were used to determine the effects of gender and age.

Results: The obtained results found that HpSA-LFIA achieved promising sensitivity $(93.75 \%)$ and NPV (98.00\%). However, it had poor specificity, PPV, and accuracy, respectively, 59.76\%, 31.25\%, and $65.31 \%$. LR+ \& LR- were $2.33 \%$ \& $0.1 \%$, respectively. Gender had no significance on the diagnostic parameters of HpSA-LFIA. Age groups had irrelevant sensitivity; however, specificity was significantly higher in patients over 45 years.

Conclusion: It was concluded that HpSA-LFIA was not accurate enough to be the sole test for diagnosis and needs other confirmatory tests in case of positive conditions.

Keywords: HpSA; H. pylori; diagnostic values; sensitivity; specificity; accuracy; PPV; NPV

\section{How to Use This Template}

The template details the sections that can be used in a manuscript. Note that each section has a corresponding style, which can be found in the "Styles" menu of Word. Sections that are not mandatory are listed as such. The section titles given are for articles. Review papers and other article types have a more flexible structure.

Remove this paragraph and start section numbering with 1 . For any questions, please contact the editorial office of the journal or support@mdpi.com.

\section{Introduction}

Helicobacter pylori infection is characterized by chronic gastritis, peptic ulcer, gastric cancer as mucosa-associated lymphoid tissue (MALT) lymphoma, and extra gastric disorders such as atherosclerosis and skin lesions [1]. H. pylori infection is one of the most common 
public health problems, affecting approximately $50 \%$ of the world's population [2]. Identifying $H$. pylori infection is crucial for an appropriate selection of the disease therapy and eradication follow-up protocols. Invasive and non-invasive assays could diagnose the infection. Gastric biopsies are used in the invasive procedure (endoscopy) to detect $H$. pylori using a rapid urease test, histopathology, PCR, and culture. The presence of active H. pylori infection could be detected via urea breath test (UBT) and stool antigen tests. Serological tests detected anti-H. pylori antibodies, indicating that the patient had a passive $H$. pylori infection [3].

The invasive approaches for diagnosis are costly, time-consuming, and generally require more than one confirmatory test. On the other hand, the non-invasive approaches could detect $H$. pylori active and passive infections [2]. Many studies reported that the stool antigen assays are highly sensitive and specific [4]. The European Helicobacter pylori study group has recommended the stool antigen test as a non-invasive test for diagnosis [5]. The non-invasive methods as $H$. pylori stool antigen-lateral flow immunochromatography assay (HpSA-LFIA) [6-8] and enzyme immunoassays (EIA) -as semiquantitative ELISA- are used for stool antigen detection [9, 10]. HpSA-LFIA, an office-based test, is preferred due to its fastness, applicability, reliability, and long shelf life at room temperature (12-24 months) [11]. A comparison of commonly used HpSA-ELISA and HpSA-LFIA reported that the latter had reasonable specificity and sensitivity in children [8].

The meta-analysis studies suggested the superiority of monoclonal antibody-based stool antigen tests compared to polyclonal antibody-based ones in the initial diagnosis of $H$. pylori infection. According to the European Guidelines, monoclonal antibody-based tests and UBT are the most recommended non-invasive assays for monitoring the success or failure of eradication treatment $[3,12]$. Although the UBT is an accurate non-invasive test, it is comparatively costly and depends upon mass spectrometric analysis, which is not convenient for small centers with limited resources in developing countries [13]. Moreover, certain studies accounted for the lower specificity of the UBT in young ages. Falsepositive results may be attributed to urease-producing bacteria from the oral cavity in non-infected children [14].

HPSA-LFIA could be used as an alternative to UBT to diagnose primary infection of $H$. pylori, especially in developing countries. LFIA is faster than the conventional ELISA, which takes more than two hours to be performed [8, 15-18].

Several HpSA-LFIA strips are currently commercially available for the diagnosis of $H$. pylori infection. It is a qualitative test used either to detect anti- $H$. pylori antibodies or $H$. pylori antigens in clinical samples. Both are intended to aid in diagnosing infection in adult patients and follow up the infection eradication [2].

This study aimed to evaluate the diagnostic values of polyclonal-based HpSA-LFIA, the most commercially available assay in Egypt. The evaluation was established by using a reference test, monoclonal-based, and semiquantitative double sandwich HpSA-ELISA. Statistical analysis was performed to find the sensitivity, specificity, accuracy, PPV, NPV, LR+, and LR- in different genders and ages.

\section{Materials and Methods}

\subsection{Samples:}

Random stool samples were properly collected from 200 gastric patients (80 males and 120 females) from the end of 2019 to summer 2020. The participants ranged in age from 3 to 55 years old. The collected samples were divided into three age groups: 13 patients aged 3 to 18,136 patients aged 18 to 45, and 47 patients aged above 45 years. According to their physician recommendations, these patients went to the clinical laboratory for 
rapid stool antigen detection with written and assigned consent. This study was approved by the Research Ethics Committee process number (HAM00116).

The sample size was calculated with a power of $80 \%$ (https://www.calculator.net/sample-size-calculator.html). The samples were collected from 3 Egyptian governorates: Menofia, Benha, and Giza. None of the patients had taken any antibiotics, antacids or proton pump inhibitors (PPIs) one month before sample collection. The stool samples were tested immediately after collection for LFIA and preserved at $-20^{\circ} \mathrm{C}$ until the ELISA test performance. The samples were transferred to the Microbiology department, Faculty of Veterinary Medicine, Cairo University, Giza, Egypt, where the HpSA-ELISA was performed (during the same week of collection).

H. pylori stool antigen-Lateral flow immunochromatography assay (HpSA-LFIA):

Rightsign ${ }^{\circledR}$ H. pylori stool antigen rapid test (Hangzhou Biotest Biotech Co., Ltd, Hangzhou, China. Cat.No. R0192c) was applied to the samples according to its pamphlet instructions. The product features were $96.7 \%$ sensitivity, $93.8 \%$ specificity (https://ctkbiotech.com/product/h-pylori-ag-rapid-test-ce/). This assay used polyclonal anti-H. pylori antibodies. The procedures were performed in three steps, as follows:

1. Sample preparation by stabbing $50 \mathrm{mg}$ of the stool sample from three different sites or $80 \mu \mathrm{l}$ in diarrheal samples, then the samples were transferred into an extraction buffer.

2. A few drops (about $80 \mu \mathrm{l}$ ) of the extracted sample were transferred into the LFIA cassette.

3. The results were read after ten minutes of incubation at room temperature.

The results were read by different specialists to avoid individual error.

2.2. H. pylori stool antigen-enzyme immunoassay (HPSA-ELISA):

H. pylori Stool Antigen ELISA kit was used in the study for each sample (FORESIGHT® H. pylori antigen EIA test kit, Acon laboratories Inc., 10125 Mesa Rim Road, San Diego, CA 92121, USA, REF 1231-1231). Performance characteristics were $98.6 \%$ sensitivity (95\% CI : $92.4-100.0 \%), 95.4 \%$ specificity (95\% CI : $90.3-98.3 \%$ ) and overall agreement $96.5 \%$ (95\% CI : 93.0-98.6\%). The procedures were applied according to the manufacturer's instructions. It is a semiquantitative, containing $\mathrm{H}$. pylori Antigen standard set $(0,5,10,25$, 50 , and $100 \mathrm{ng} / \mathrm{ml}$ ). HpSA-ELISA is a double sandwich assay in which the microplates were coated with monoclonal anti-H. pylori antibodies. The plate optical densities (ODs) were detected by ELISA reader at $450 \mathrm{~nm}$ wavelength. The optical densities of each sample were calculated. The results were obtained by calculating the mean absorbance value of reference standards, specimens, controls, and patient samples. A standard curve was constructed by patting the mean absorbance obtained from each reference standard ( $\mathrm{Y}$ axis) against its concentration in $\mathrm{ng} / \mathrm{ml}$ (X-axis). The absorbance values were used to determine the corresponding concentration of $\mathrm{H}$. pylori antigen in $\mathrm{ng} / \mathrm{ml}$. Sample concentrations greater than $100 \mathrm{ng} / \mathrm{ml}$ were considered out of the range of the standard curve (borderline). The interpretation was considered positive if the antigen concentration was more than $20 \mathrm{ng} / \mathrm{ml}$ and negative if the concentration was less than $15 \mathrm{ng} / \mathrm{ml}$. The readings between $15-20 \mathrm{ng} / \mathrm{ml}$ were considered suspicious, and the sample should be repeated later. 


\subsection{Statistical analysis:}

The ELISA test results were used to define H. pylori status. Borderline results $(n=4)$ were then excluded. For all patients and each sex-age group, sensitivity, specificity, positive predictive value (PPV), negative predictive value (NPV), positive likelihood ratio $(\mathrm{LR}+)$, negative likelihood ratio (LR-), accuracy, disease prevalence, and their confidence intervals $(95 \% \mathrm{CI})$ were calculated against the defined $\mathrm{H}$. pylori status using MedCalc software version 20.008 (www.medcalc.org/calc/diagnostic_test.php). Chi-square test was used to analyze the effects of age and gender on the test performance of HpSALFIA. All p-values less than 0.05 were considered statistically significant. Data were presented as the mean \pm standard error of the mean (SE). Figures were plotted, and statistical analyses were performed using SigmaPlot v14.0 (Systat Software, San Jose, CA, USA).

\section{Results}

Lateral flow assay of 196 stool samples revealed 100 negative and 96 positive results (4 samples were borderline). The readings of the ELISA kit reported 32 positive results $(16.33 \%)$.

The results comparison between LFIA and ELISA showed that 30 samples were true positive (TP). Sixty-six samples were positive LFIA but negative for ELISA (FP). Only two samples were negative LFIA, but positive ELISA (FN) and 98 samples were negative for both (TN). The distribution of TP, FP, FN, and TN among different genders and ages was demonstrated in Table 1 . The disease prevalence rate and the statistical diagnostic values of the HpSA-LFIA in comparison with the HpSA-ELISA (the reference test in this study) were reported in Tables 2 and 3.

The boxplots of age distributions of males and females tested positive or negative by HPSA-LFIA were demonstrated in Figure 1. These boxplots showed the age mean and median of the tested males and females. The age of patients who tested negative ranged between 3 and 55 years ( $37.19 \pm 1.61$ years; mean \pm SE) for males and ranged from 3 to 55 years ( $37.63 \pm 0.99$ years) for females. While the age of patients who tested positive ranged between 3.5 and 45 years $(28.69 \pm 3.49$ years) for males and ranged from 4 to 54 years (33.14 \pm 4.00 years) for females. The probability (P-value) of gender effect on the test's sensitivity and specificity was 0.5882 and 0.2861 , while the P-values of the age effect on sensitivity and specificity were 0.1911 and 0.0183 , respectively. 
3.2. Figures, Tables:

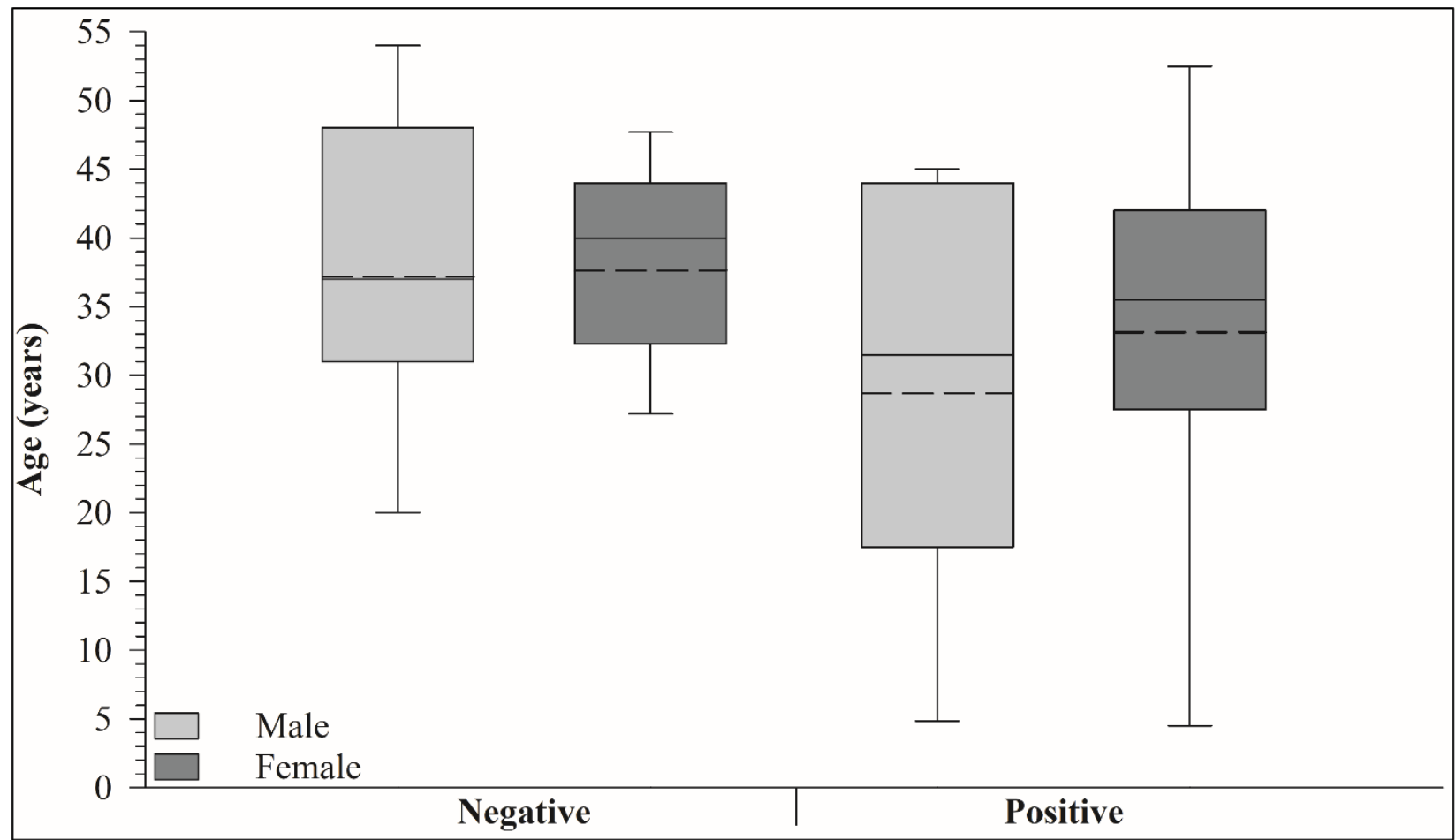

Figure 1: Boxplots of age distributions of males and females tested positive or negative by lateral flow immunoassay tests. Dashed lines indicate mean while solid lines indicate median. Four individuals were removed as they were borderline (3 males; 1 female). 


\begin{tabular}{|c|c|c|c|c|c|c|c|c|c|c|c|c|c|c|}
\hline & \multicolumn{14}{|c|}{ LFIA } \\
\hline & & & \multicolumn{4}{|c|}{ Positive } & \multicolumn{7}{|c|}{ Negative } & \multirow[b]{3}{*}{ Tota } \\
\hline & & & Male & & & Female & & & Male & & & Female & & \\
\hline & & $0-18 y$ & $20-45 y$ & $>45 y$ & $3-18 y$ & $20-45 y$ & $>45 y$ & $0-18 y$ & $20-45 y$ & $>45 y$ & $0-18 y$ & $20-45 y$ & $>45 y$ & \\
\hline \multirow[t]{3}{*}{ ELISA } & Positive & 3 & 11 & 3 & 2 & 8 & 3 & 1 & 0 & 0 & 0 & 1 & 0 & 32 \\
\hline & Negative & 2 & 20 & 8 & 3 & 29 & 4 & 0 & 18 & 11 & 2 & 49 & 18 & 164 \\
\hline & Total & 5 & 31 & 11 & 5 & 37 & 7 & 1 & 18 & 11 & 2 & 50 & 18 & 196 \\
\hline
\end{tabular}

Table 2 The diagnostic values of LFA in comparison to ELISA showing the parameters in all patients and separate gender

\begin{tabular}{llll}
\hline & \multicolumn{1}{c}{ All } & \multicolumn{1}{c}{ Male } & \multicolumn{1}{c}{ Female } \\
\hline $\mathbf{N}(\%)$ & $196(100.0)$ & $77(39.29)$ & $119(60.71)$ \\
Sensitivity, \% & 93.75 & 94.44 & 92.86 \\
$(95 \%$ CI $)$ & $(79.19-99.23)$ & $(72.71-99.86)$ & $(66.13-99.82)$ \\
Specificity, \% & 59.76 & 49.15 & 65.71 \\
$(95 \%$ CI $)$ & $(51.83-67.33)$ & $(35.89-62.5)$ & $(55.81-74.7)$ \\
PPV, $\%$ & 31.25 & 36.17 & 26.53 \\
$(95 \%$ CI $)$ & $(26.99-35.86)$ & $(30.1-42.72)$ & $(21.07-32.82)$ \\
NPV, $\%$ & 98.00 & 96.67 & 98.57 \\
$(95 \%$ CI) & $(92.72-99.47)$ & $(80.92-99.5)$ & $(91.22-99.78)$ \\
Accuracy, \% & 65.31 & 59.74 & 68.91 \\
$(95 \%$ CI) & $(58.19-71.95)$ & $(47.94-70.77)$ & $(59.77-77.07)$ \\
Disease prevalence, \% & 16.33 & 23.38 & 11.76 \\
$(95 \%$ CI) & $(11.44-22.26)$ & $(14.48-34.41)$ & $(6.58-18.95)$ \\
LR+ & 2.33 & 1.86 & 2.71 \\
$(95 \%$ CI) & $(1.89-2.86)$ & $(1.41-2.44)$ & $(2.00-3.66)$ \\
LR- & 0.10 & 0.11 & 0.11 \\
$(95 \%$ CI) & $(0.03-0.40)$ & $(0.02-0.77)$ & $(0.02-0.72)$
\end{tabular}


Total positive of the

reference method, $\mathrm{n}\left(\%^{2}\right)$

$32(16.33)$

$18(23.38)$

$14(11.76)$

Total positive, $\mathrm{n}\left(\%^{2}\right)$

96 (48.98)

47 (61.04)

49 (41.18)

False negative, $\mathrm{n}\left(\%^{2}\right)$

2 (1.02)

$1(1.30)$

$1(0.84)$

False positive, $\mathrm{n}\left(\%^{2}\right)$

$66(33.67)$

30 (38.96)

$36(30.25)$

${ }^{1}$ Calculated as \% of 196 individuals. ${ }^{2}$ Calculated as \% of column total (N). 95\% CI, 95\% confidence intervals; PPV, positive predictive value; NPV, negative predictive value; LR+, likelihood ratio for positive test result. LR-, likelihood ratio for negative test result. Borderline results were excluded. 


\begin{tabular}{|c|c|c|c|c|c|c|c|c|c|}
\hline & \multicolumn{3}{|c|}{ All } & \multicolumn{3}{|c|}{ Male } & \multicolumn{3}{|c|}{ Female } \\
\hline & 0 - 18 years & 18 - 45 years & $>45$ years & 0 - 18 years & 18 - 45 years & $>45$ years & 0 - 18 years & 18 - 45 years & $>45$ years \\
\hline $\mathbf{N}\left(\%^{1}\right)$ & $13(6.63)$ & $136(69.39)$ & $47(23.98)$ & $6(3.06)$ & $49(25.00)$ & $22(11.22)$ & $7(3.57)$ & 87 (44.39) & $25(12.76)$ \\
\hline Sensitivity, $\%$ & 83.33 & 95.00 & 100.0 & 75.00 & 100.0 & 100.0 & 100.0 & 95.00 & 95.00 \\
\hline$(95 \% \mathrm{CI})$ & (35.88-99.58) & (75.13-99.87) & $(54.07-100.0)$ & (19.41-99.37) & $(71.51-100.0)$ & $(29.24-100.0)$ & $(15.81-100.0)$ & $(51.75-99.72)$ & $(29.24-100)$ \\
\hline Specificity, $\%$ & 28.57 & 57.76 & 70.73 & 0.00 & 47.37 & 57.89 & 40.00 & 62.82 & 81.82 \\
\hline$(95 \% \mathrm{CI})$ & $(3.67-70.96)$ & $(48.24-66.87)$ & $(54.46-83.87)$ & $(0.00-84.19)$ & (30.98-64.18) & $(33.50-79.75)$ & $(5.270-85.34)$ & $(51.13-73.50)$ & $(59.72-94.81)$ \\
\hline PPV, \% & 50.00 & 27.94 & 33.33 & 60.00 & 35.48 & 27.27 & 40.00 & 21.62 & 42.86 \\
\hline$(95 \% \mathrm{CI})$ & (35.67-64.33) & $(23.46-32.92)$ & $(23.7-44.59)$ & $(46.00-72.54)$ & $(28.92-42.65)$ & $(18.12-38.85)$ & $(24.58-57.69)$ & $(16.01-28.53)$ & $(23.61-64.54)$ \\
\hline $\begin{array}{l}\text { NPV, \% } \\
(95 \% \mathrm{CI})\end{array}$ & $\begin{array}{l}66.67 \\
(19.07-94.44)\end{array}$ & $\begin{array}{l}98.53 \\
(90.79-99.78)\end{array}$ & 100.0 & 0.00 & 100.0 & 100.0 & 100.0 & $\begin{array}{l}98.00 \\
(88.45-99.68)\end{array}$ & 100.0 \\
\hline Accuracy, $\%$ & 53.85 & 63.24 & 74.47 & 50.00 & 59.18 & 63.64 & 57.14 & 65.52 & 84.00 \\
\hline$(95 \% \mathrm{CI})$ & $(25.13-80.78)$ & $(54.55-71.33)$ & $(59.65-86.06)$ & $(11.81-88.19)$ & (44.21-73.00) & $(40.66-82.80)$ & $(18.41-90.10)$ & $(54.56-75.39)$ & $(63.92-95.46)$ \\
\hline Disease prevalence, $\%$ & 46.15 & 14.71 & 12.77 & 66.67 & 22.45 & 13.64 & 28.57 & 10.34 & 12.00 \\
\hline$(95 \% \mathrm{CI})$ & $(19.22-74.87)$ & $(9.22-21.79)$ & $(4.83-25.74)$ & $(22.28-95.67)$ & $(11.77-36.62)$ & $(2.91-34.91)$ & (3.67-70.96) & $(4.84-18.73)$ & $(2.55-31.22)$ \\
\hline LR+ & 1.17 & 2.25 & 3.42 & 0.75 & 1.90 & 2.38 & 1.67 & 2.39 & 5.50 \\
\hline$(95 \% \mathrm{CI})$ & $(0.65-2.10)$ & $(1.78-2.85)$ & $(2.12-5.50)$ & $(0.43-1.32)$ & $(1.41-2.57)$ & $(1.4-4.02)$ & $(0.81-3.41)$ & $(1.65-3.46)$ & $(2.27-13.35)$ \\
\hline $\begin{array}{l}\text { LR- } \\
(95 \% \mathrm{CI})\end{array}$ & $\begin{array}{l}0.58 \\
(0.07-4.95)\end{array}$ & $\begin{array}{l}0.09 \\
(0.01-0.59)\end{array}$ & 0.00 & -- & 0.00 & 0.00 & 0.00 & $\begin{array}{l}0.18 \\
(0.03-1.13)\end{array}$ & 0.00 \\
\hline $\begin{array}{l}\text { Total positive of the } \\
\text { reference method, } n\left(\%^{2}\right)\end{array}$ & $6(46.15)$ & $20(14.71)$ & $6(12.77)$ & $4(66.67)$ & $11(22.45)$ & $3(13.64)$ & $2(28.57)$ & $9(10.34)$ & $3(12.00)$ \\
\hline Total positive, $\mathrm{n}\left(\%^{2}\right)$ & $10(76.92)$ & $68(50.00)$ & $18(38.30)$ & $5(83.33)$ & $31(63.27)$ & $11(50.00)$ & $5(71.43)$ & $37(42.53)$ & $7(28.00)$ \\
\hline False negative, $\mathrm{n}\left(\%^{2}\right)$ & $1(7.69)$ & $1(0.74)$ & $0(0.00)$ & $1(16.67)$ & $0(0.00)$ & $0(0.00)$ & $0(0.00)$ & $1(1.15)$ & $0(0.00)$ \\
\hline False positive, $\mathrm{n}\left(\%^{2}\right)$ & $5(38.46)$ & $49(36.03)$ & $12(25.53)$ & $2(33.33)$ & $20(40.82)$ & $8(36.36)$ & $3(42.86)$ & $29(33.33)$ & $4(16.00)$ \\
\hline
\end{tabular}

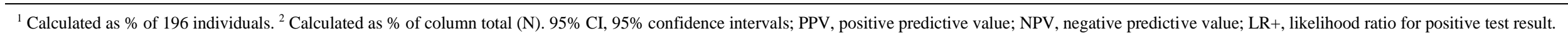

LR-, likelihood ratio for negative test result. Borderline results were excluded. 


\section{Discussion}

This study evaluates and reports the diagnostic values (Se, Sp, PPV, NPV, LR+, LR-, and accuracy) of Egypt's most common non-invasive test. Rightsign ${ }^{\circledR}$ Helicobacter pylori stool Antigen rapid test was compared to Foresight ${ }^{\circledR}$ semiquantitative HpSA-ELISA. The latter is considered a specific and sensitive test as it could detect low antigen concentrations $(0.5 \mathrm{ng} / \mathrm{ml})$.

The results of HpSA-LFIA showed poor specificity (59.76\%) but gave a good sensitivity $(93.75 \%)$. These findings did not match the product features of the Rightsign H. pylori Ag rapid test, as our results reported a dramatic drop in test specificity. The precision of the HpSA-LFIA indicated un-satisfactory PPV (31.25\%). Nevertheless, it had an acceptable NPV (98.00\%). The accuracy of the HpSA-LFIA (65.31\%) was not promising to confirm the diagnosis but sufficient to negate the disease.

The likelihood ratio (LR) assesses the utility of the LFIA and how likely the patient is infected. HpSA-LFIA had low LR+ (2.33), which indicated a low possibility of true positive cases. On the opposite side, it had a reliable LR- (0.10), which implied a low possibility of false-negative cases.

The results of HpSA-LFIA reported no statistical significance of gender in sensitivity (t4 $=0.59, \mathrm{P}=0.5882)$ or specificity $(\mathrm{t} 4=1.23, \mathrm{P}=0.2861)$.

The highest HpSA-LFIA diagnostic values were obtained in elders over 45 years old. In young less than 18 years, PPV, false positive, and false negative were the highest values. The specificity in young was modest (Table 3), as mentioned by Frenck et al., who found that the specificity was significantly lower among Egyptian children under six years [19]. Age groups had no significant effect on sensitivity ( $F 2.6=2.21, p=0.1911)$. However, specificity was significantly higher in elders (over 45 years) than younger (less than 18 years) $(\mathrm{F} 2.6=8.42, \mathrm{p}=0.0183)$.

The previous results agree with da Silva, Kato et al., and others who recorded that HpSA-LFIA presented 52.5-94.6\% sensitivity, 55.5-98.4\% specificity, and 98.4\% NPV; however, disagree in PPV value (94.6\%) [1,2,8,16-18]. Other similar studies used an insufficient sample size (65 stool samples) with the ImmunoCard STAT! HpSA assay, obtained $77.8 \%$ sensitivity, $79.3 \%$ specificity, $82.4 \%$ PPV, and 74.2\% NPV [20].

Our findings did not concede with Karakus, Salih, and Kato et al., who reported that the HpSA-LFIA was valid for H. pylori infection diagnosis in children and adolescents, with comparable results to ELISA. They suggested that HpSA-LFIA had high accuracy for all age groups with $\mathrm{Se} \%$ of $93 \%$ and $\mathrm{Sp} \%$ of $91 \%$. They found that in a 5 -years followup study performed in adults, the HpSA-LFIA showed a sensitivity of $93 \%$ and a specificity of 100\% [8]. Karakus and Salih revealed that the sensitivity was $90-100 \%$ (average $95 \%$ ), and the specificity was $80-100 \%$ (average $96 \%$ ) [2]. In another study that recruited 91 patients, the sensitivity of the H. pylori stool antigen test was $73.9 \%$, and the specificity was $86.7 \%$ [21]. Our results also did not trust the assessment of the pre-and posteradication diagnostic values of HpSA-LFIA compared to HpSA ELISA in children, which found that sensitivity, specificity, PPV, and NPV for the HPSA-LFIA were 94.6, 98.4, 94.6, and 98.4\%, respectively [18]. Evaluation of diagnostic accuracy of HpSA-LFIA (LINEAR Chemical, Barcelona, Spain) in 109 children with abdominal symptoms (age range, $5-17$ years; mean, 12.1 ) obtained $65.00 \%$ sensitivity and $92.3 \%$ specificity [22]. An evaluation of HpSA-LFIA in symptomatic children revealed a sensitivity of $88.9 \%$ (95\% $C I=77.3-96.3)$ and a specificity of $94.0 \%(95 \% C I=88.1-97.7)$. There was no age-dependency of the stool test results [7, 23]. 
Spatial differences perhaps affect the results of HpSA-LFIA and might interpret the vast diversity in the diagnostic values, as it was an imported kit. This finding was supported by Makristathis et al., who reported that HPSA-LFIA prepared with polyclonal anti-H. pylori antibodies varied in sensitivity results, $96.6 \%$ in Brazilian children, $91.5 \%$ in Italian children, and $67 \%$ in Italian adults. However, the Se\% in Egyptian adults was $57.7 \%$ [9]. H. pylori mediates natural transformation and mechanisms of bacterial DNA horizontal gene transfer, which maintain a high level of genetic variability [24]. H. pylori has a higher mutation rate than most bacteria [25].

Twenty-two studies (including 2,499 patients) evaluated the monoclonal stool antigen tests before eradication therapy. Pooled sensitivity, specificity, LR+, and LR- were: $94 \%$ (95\% CI $=93-95 \%), 97 \%$ (95\% CI = 96-98\%), 24 (95\% CI = 15-41), and 0.07 (95\% CI = $0.04-$ $0.12)$, respectively. The accuracy of both monoclonal and polyclonal stool antigen tests was evaluated together in 13 pretreatment studies, and higher pooled sensitivity was demonstrated with the monoclonal technique (95\% vs. 83\%). Twelve studies (including 957 patients) assessed the monoclonal stool antigen tests to confirm eradication after therapy. Pooled Sensitivity, Specificity, LR+, and LR- were 93\% (95\% CI $=89-96 \%), 96 \%$ $(95 \% \mathrm{CI}=94-97 \%), 17(95 \% \mathrm{CI}=12-23)$, and $0.1(95 \% \mathrm{CI}=0.07-0.15)$, respectively. Both tests were evaluated together in 8 post-treatment studies, and once more, the monoclonal technique showed a higher sensitivity (91\% vs. 76\%) [12]. Most literature concluded that better results were obtained for invasive vs. non-invasive tests. For a more accurate diagnosis, it is advisable not to solely rely on non-invasive methods of $\mathrm{H}$. pylori diagnosis [21].

The strength points of our study were using a suitable and calculated sample size of diseased patients. It is noteworthy that the sample size was more than any study in literature [8,15-17, 19-21]. An accurate test (ELISA) was used as a reference test to evaluate HpSA-LFIA. Moreover, several genders and ages were included in the study, and various statistical parameters were calculated to measure the diagnostic values and utility. However, more comparisons with other invasive and non-invasive tests and larger sample sizes from more Egyptian governorates are highly recommended. These points will be considered in our future studies.

\section{Conclusions}

HPSA-LFIA in our country is a highly sensitive test with low specificity and low accuracy to be the sole test for diagnosis. The test was intended to be used as a screening test and provided a preliminary result which was not enough for precision and final diagnosis. There is an urgent demand for developing an accurate, rapid monoclonal antibody based LFIA from local H. pylori isolates.

Author Contributions: Sh. A.: The idea constructor, sample and data collection and ELISA, manuscript drafting, and revision.

H. A.: Statistical analysis, manuscript drafting, and revision.

W. H.: Samples collections, LFIA

N. N.: ELISA

K.Sh: Manuscript drafting and revision.

For research articles with several authors, a short paragraph specifying their individual contributions must be provided. The following statements should be used "Conceptualization, Sh.A. and W.H..; methodology, Sh.A and N.N..; software, H.A..; validation, Sh. A., W.H., and H.A. .; formal analysis, H.A.; investigation, Sh. A..; resources, Sh. A. and W.H..; data curation, Sh. A..; writingoriginal draft preparation, Sh. A.; writing - review and editing, Sh. A. and K. Sh.; visualization, Sh. A.; supervision, Sh. A.; project administration, Sh.A.; funding acquisition, W.H. All authors have read and agreed to the published version of the manuscript.

Funding: This research received no external funding. 
Institutional Review Board Statement: This study was approved by the Research Ethics Committee process number (HAM00116).

Informed Consent Statement: Informed consent was obtained from all subjects involved in the study.

Conflicts of Interest: The authors declare no conflict of interest.

\section{References}

1. Den Hoed CM, Kuipers EJ. Helicobacter pylori Infection. Hunter's Trop Med Emerg Infect Dis Ninth Ed. 2012;347(15):437441. doi:10.1016/B978-1-4160-4390-4.00041-2

2. Karakus C, Salih BA. Comparison of the lateral flow immunoassays (LFIA) for the diagnosis of Helicobacter pylori infection. J Immunol Methods. 2013;396(1-2):8-14. doi: 10.1016/j.jim.2013.08.010

3. Ricci C, Holton J, Vaira D. Diagnosis of Helicobacter pylori: Invasive and non-invasive tests. Best Pract Res Clin Gastroenterol. 2007;21(2):299-313. doi: 10.1016/j.bpg.2006.11.002

4. Vaira D, Vakil N. Blood, urine, stool, breath, money, and Helicobacter pylori. Gut. 2001;48(3):287-289. doi:10.1136/gut.48.3.287

5. Malfertheiner P, Mégraud F, O'Morain C, et al. Current concepts in the management of Helicobacter pylori infection The Maastricht 2-2000 Consensus Report. Aliment Pharmacol Ther. 2002;16(2):167-180. doi:10.1046/j.1365-2036.2002. 01169.x

6. Braden B, Posselt H, Ahrens P, Kitz R, Dietrich CF, Caspary WF. New Immunoassay in Stool Provides an Accurate Noninvasive Diagnostic Method for Helicobacter pylori Screening in Children. Pediatrics. July 2000 ; 106 (1) 115-117. doi:10.1542/peds.106.1.115

7. Konstantopoulos N, Rüssmann H, Tasch C, et al. Evaluation of the Helicobacter pylori stool antigen test (HpSA) for detection of Helicobacter pylori infection in children. Am J Gastroenterol. 2001;96(3):677-683. doi:10.1016/S00029270(00)02396-0

8. Kato S, Ozawa K, Okuda M, et al. Multicenter comparison of rapid lateral flow stool antigen immunoassay and stool antigen enzyme immunoassay for the diagnosis of Helicobacter pylori infection in children. Helicobacter. 2004;9(6):669673. doi:10.1111/j.1083-4389.2004. 00279.x

9. Makristathis A, Hirschl AM, Lehours P, Mégraud F. Diagnosis of Helicobacter pylori infection. Helicobacter. 2004;9 Suppl 1:7-14. doi: 10.1111/j.1083- 4389.2004.00254. x.

10. Oderda G, Rapa A, Ronchi B, et al. Detection of Helicobacter pylori in stool specimens by non-invasive antigen enzyme immunoassay in children: multicentre Italian study Effect of hormone replacement therapy on the pathological stage of breast cancer: population based, cross sectional st. Bmj. 2000;320(7231):347-8. 10.1136/bmj.320.7231.347.

11. Ngom B, Guo Y, Wang X, Bi D. Development and application of lateral flow test strip technology for detection of infectious agents and chemical contaminants: A review. Anal Bioanal Chem. 2010;397(3):1113-1135. doi:10.1007/s00216-010-3661-4

12. Gisbert JP, De La Morena F, Abraira V. Accuracy of monoclonal stool antigen test for the diagnosis of H.pylori infection: A systematic review and meta-analysis. Am J Gastroenterol. 2006;101(8):1921-1930. doi:10.1111/j.1572-0241.2006. 00668.x

13. Kato M, Saito M, Fukuda S, et al. 13C-Urea breath test, using a new compact frared spectrophotometer: comparison with mass spectrometry. nondispersive isotope-selective indoi:10.1007/s00535-003-1357-7

14. Imrie C, Rowland M, Bourke B, Drumm B. Limitations to carbon 13-labeled urea breath testing for Helicobacter pylori in infants. J Pediatr. 2001 Nov;139(5): 734-7.doi: $\quad$ 10.1067/mpd.2001.118398. PMID: 11713455.

15. Da Silva JMK, Villares CA, Monteiro M do S, Colaúto C, dos Santos AF, Mattar R. Validation of a rapid stool antigen test for diagnosis of Helicobacter pylori Infection. Rev Inst Med Trop Sao Paulo. 2010;52(3):125-128. doi:10.1590/S003646652010000300002

16. Blanco S, Forné M, Lacoma A, et al. Comparison of stool antigen immunoassay methods for detecting Helicobacter pylori infection before and after eradication treatment. Diagn Microbiol Infect Dis. 2008;61(2):150-155. doi: 10.1016/j.diagmicrobio.2008.01.003

17. Krausse R, Müller G, Doniec M. Evaluation of a rapid new stool antigen test for diagnosis of Helicobacter pylori infection in adult patients. J Clin Microbiol. 2008;46(6):2062-2065. doi:10.1128/JCM.02387-07

18. Yang HR, Seo JK. Helicobacter pylori stool antigen (HpSA) tests in children before and after eradication therapy: Comparison of rapid immunochromatographic assay and HpSA ELISA. Dig Dis Sci. 2008;53(8):2053-2058. doi:10.1007/s10620-0070131-8

19. Frenck RW, Fathy HM, Sherif M, et al. Sensitivity and specificity of various tests for the diagnosis of Helicobacter pylori in Egyptian children. Pediatrics. 2006;118(4) e1195-202. doi:10.1542/peds.2005-2925

20. Kaklikkaya N, Akdogan RA, Ozgur O, et al. Evaluation of a new rapid lateral flow chromatography test for the diagnosis of Helicobacter pylori. Saudi Med J. 2006;27(6):799-803. PMID: 16758038

21. Khalifehgholi M, Shamsipour F, Ajhdarkosh H, et al. Comparison of five diagnostic methods for Helicobacter pylori. Iran J Microbiol. 2013;5(4):396-401. PMID: 25848511 
22. Kuloğlu Z, Kansu A, Kirsaçlioğlu CT, et al. A rapid lateral flow stool antigen immunoassay and 14C-urea breath test for the diagnosis and eradication of Helicobacter pylori infection in children. Diagn Microbiol Infect Dis. 2008;62(4):351-356. doi: 10.1016/j.diagmicrobio.2008.07.006

23. Kato S, Ozawa K, Okuda M, et al. Accuracy of the stool antigen test for the diagnosis of childhood Helicobacter pylori infection: A multicenter Japanese study. Am J Gastroenterol. 2003;98(2):296-300. doi:10.1016/S0002-9270(02)05931-2

24. Chmiela M, Kupcinskas J. Review: Pathogenesis of Helicobacter pylori infection. Helicobacter. 2019;24 Suppl 1(Suppl Suppl 1): e12638. doi:10.1111/hel.12638

25. Thorpe H., Tourrette E., Yahara K., et al. Repeated out-of-Africa expansions of Helicobacter pylori driven by replacement of deleterious mutations. bioRxiv .2021. https://doi.org/10.1101/2021.06.05.447065 